\title{
ON HEMINEARNESS SPACES
}

\author{
MARYAM SHAYEGAN HASTINGS ${ }^{1}$
}

\begin{abstract}
The concept of nearness was introduced by $H$. Herrlich [4'], where nearness refers to collections of sets. Nearness spaces have proven to be an extremely useful generalization of a wide class of topological structures.
\end{abstract}

A category which contains $N E A R$ as a subcategory is the category $H-N E A R$, of heminearness spaces. It arises naturally while studying products of nearness spaces. A nice embedding theorem further motivated us to make a detailed study of this category. H. Herrlich [4'] has described a process by which one can construct completions for nearness spaces. $H-N E A R$ is the only supercategory of $N E A R$ known to us where this process of completion can be imitated satisfactorily.

In §I we define heminearness, give some examples, describe initial, final, product, coproduct, subobject, quotient object, and completion for heminearness spaces.

In §II we introduce the concept of preclosure, semiclosure, and hemiclosure. We show that any heminearness space can be embedded as a subspace of a closure heminearness space.

I. Let $X$ be a set and $\xi \subset P^{2} X$ and consider the following axioms:

(N1) If $\mathfrak{L} \in \xi$ and $\mathfrak{A}$ corefines $\mathfrak{R}$ (i.e., for each $A \in \mathfrak{A}$ there exists a $B \in \mathfrak{L}$ such that $B \subset A$ ) then $\mathfrak{A} \in \xi$.

(N2) If $\cap \mathfrak{A} \neq \varnothing$, then $\mathfrak{A} \in \xi$.

(N3) $\varnothing \neq \xi \neq P^{2} X$.

(N4) If $\mathfrak{A} \vee \mathfrak{L}=\{A \cup B \mid A \in \mathfrak{A}, B \in \mathfrak{L}\} \in \xi$, then $\mathfrak{A} \in \xi$ or $\mathfrak{L} \in \xi$.

(N5) If $\mathrm{Cl}_{\xi} \mathfrak{A} \in \xi$, then $\mathfrak{A} \in \xi .\left(\mathrm{Cl}_{\xi} A=\{x \in X \mid\{\{x\}, A\} \in \xi\}\right.$ and $\mathrm{Cl}_{\xi} \mathfrak{A}=$ $\left\{\mathrm{Cl}_{\xi} A \mid A \in \mathfrak{A}\right\}$.)

1. Definition [5]. $(X, \xi)$ is called a prenearness [seminearness, nearness] space if and only if $\xi$ satisfies (N1)-(N3) [(N1)-(N4),(N1)-(N5)]. These spaces were introduced by $\mathrm{H}$. Herrlich [5].

2. Definition [5]. If $(X, \xi)$ and $(Y, \eta)$ are prenearness spaces, then a function $f$ : $(X, \xi) \rightarrow(Y, \eta)$ is called a nearness-preserving map if and only if $\mathfrak{A} \in \xi$ implies that $f(\mathfrak{U}) \in \eta$.

Received by the editors January 5, 1982 and, in revised form, May 6, 1982.

1980 Mathematics Subject Classification. Primary 18B30, 54A05, 54B30, 54E17.

Key words and phrases. Heminearness spaces, closure heminearness spaces.

'This paper was presented at the U.S. Naval Academy Topology Conference, March 11-13, 1982. This conference was sponsored by the Department of Mathematics at the U.S. Naval Academy with support from the Office of Naval Research and the National Science Foundation. 
We consider those spaces where $\xi$ satisfies (N1)-(N3) and (N5). We call them heminearness spaces; they were first introduced by Hunsaker and Sharma [9].

We will give some examples of heminearness spaces, and describe the relation between the category of heminearness spaces and the categories of prenearness spaces and nearness spaces. Initial, final, product, coproduct, subobjects, and quotient objects are described in the category of heminearness spaces. We also describe completions for heminearness spaces.

3. Examples. (a) Let $\mathbf{R}$ denote the reals and for each $A \subseteq \mathbf{R}$ define $\mathrm{Cl} A=$ convex hull of $A$. Let $\xi=\{\mathfrak{A} \subset P \mathbf{R} \mid \cap \mathrm{Cl} \mathfrak{A} \neq \varnothing\}$. Then $\xi$ is a heminearness structure on $\mathbf{R}$ which is not a nearness structure. Note that if $A$ and $B$ are subsets of $\mathbf{R}$, $\mathrm{Cl}(A \cup B) \neq \mathrm{Cl} A \cup \mathrm{Cl} B$ in general.

(b) Let $(\mathbf{R}, \xi)$ be defined as follows: Let $(\mathbf{R}, \mathrm{Cl})$ denote the reals with the usual topology and $\xi=\{\mathfrak{A} \subset P \mathbf{R} \mid \cap \mathrm{Cl} \mathfrak{A} \neq \varnothing\}$, then $(\mathbf{R}, \xi)$ is a nearness space. Let $\eta=\left\{\mathfrak{A} \subset P(\mathbf{R} \times \mathbf{R}) \mid \operatorname{Pr}_{i} \mathfrak{A} \in \xi\right.$ for $\left.i=1,2\right\}$, where $\operatorname{Pr}_{i}: \mathbf{R} \times \mathbf{R} \rightarrow \mathbf{R}$ is the canonical projection for $i=1,2$. Then $\eta$ is a heminearness structure on $\mathbf{R} \times \mathbf{R}$ which is not a nearness structure. In particular, if $A$ and $B$ are subsets of $\mathbf{R} \times \mathbf{R}, \mathrm{Cl}(A \cup B) \neq$ $\mathrm{Cl} A \cup \mathrm{Cl} B$ in general.

The category of prenearness [seminearness, heminearness, nearness] spaces and nearness preserving maps is called P-NEAR [S-NEAR, H-NEAR, NEAR].

4. THEOREM. NEAR is a bicoreflective subcategory of H-NEAR. For any heminearness space $(X, \xi)$, the map $1_{X}:\left(X, \xi_{s}\right) \rightarrow(X, \xi)$ is the NEAR coreflection of $(X, \xi)$ where $\xi_{s}$ is defined as in [5] as follows: $\mathfrak{A} \notin \xi_{s}$ if and only if there exists $\mathfrak{A}_{1} \cdots \mathfrak{A}_{n} \notin \xi$ such that $\mathfrak{U}_{1} \vee \cdots \vee \mathfrak{A}_{n}$ corefines $\mathfrak{A}$.

5. THEOREM. H-NEAR is a bireflective subcategory of P-NEAR. For any P-Near space $(X, \xi)$, the map $1_{X}:(X, \xi) \rightarrow\left(X, \xi_{n}\right)$ is the $H$-NEAR bireflection of $(X, \xi)$, where $\xi_{n}$ is defined as in [5] as follows:

(1) $\mathrm{Cl}^{0} A=A$.

(2) $\mathrm{Cl}^{\alpha+1} A=\left\{x \in X \mid\left\{\mathrm{Cl}^{\alpha} A, \mathrm{Cl}^{\alpha}\{x\}\right\} \in \xi\right\}$.

(3) $\mathrm{Cl}^{\beta} A=\bigcup\left\{\mathrm{Cl}^{\alpha} A \mid \alpha<\beta\right\}$ if $\beta$ is a limit ordinal. $\mathrm{Cl} A=\bigcup\left\{\mathrm{Cl}^{\alpha} A \mid \alpha\right.$ is an ordinal $\} . \xi_{n}=\{\mathfrak{U} \subset P X \mid\{\mathrm{Cl} A \mid A \in \mathfrak{A}\} \in \xi\}$.

Proof. Observe that if $\alpha$ is an ordinal greater than or equal to the cardinal of $X$, then $\mathrm{Cl} A=\mathrm{Cl}^{\alpha} A$ for each $A \subset X$.

6. THEOREM. For any set $X$, the set of all heminearness structures on $X$ is a complete lattice with respect to inclusion. Especially

(1) The discrete nearness $=\{\mathfrak{U} \subset P X \mid \cap \mathfrak{U} \neq \varnothing\}$ is the smallest element.

(2) The indiscrete nearness $=\{\mathfrak{A} \subset P X \mid \varnothing \notin \mathfrak{U}\}$ is the largest element.

(3) If $\Omega$ is a set of heminearness structures on $X$, inf $\Omega=\cap \Omega$ and $\sup \Omega=(\cup \Omega)_{n}$.

7. Proposition. If $X$ is a set, $\left(X_{i}, \xi_{i}\right)_{i \in I}$ a family of heminearness spaces, then

(1) If $\left(f_{i}: X \rightarrow X_{i}\right)_{i \in I}$ is a family of maps, $\xi=\bigcap\left\{f_{i}^{-1}\left(\xi_{i}\right) \mid i \in I\right\}$ is a heminearness structure on $X$ initial with respect to $\left(X, f_{i},\left(X_{i}, \xi_{i}\right)\right)_{i \in I}$ where $f_{i}^{-1}\left(\xi_{i}\right)=\left\{\mathfrak{A} \subset P X \mid f_{i} \mathfrak{A}\right.$ $\left.\in \xi_{i}\right\}$ for each $i \in I$.

(2) If $\left(f_{i}: X_{i} \rightarrow X\right)_{i \in I}$ is a family of maps,

$$
\xi=\left(\{\mathfrak{A} \subset P X \mid \cap \mathfrak{A} \neq \varnothing\} \cup \cup\left\{f_{i}\left(\xi_{i}\right) \mid i \in I\right\}\right)_{n}
$$


is a heminearness structure on $X$ final with respect to $\left(\left(X_{i}, \xi_{i}\right), f_{i}, X\right)_{i \in I}$ where $f_{i}\left(\xi_{i}\right)=\left\{\mathfrak{U} \subset P X \mid f_{i}^{-1} \mathfrak{U} \in \xi_{i}\right\}$ for each $i \in I$.

8. COROLLARY. H-NEAR is a properly fibred topological category.

9. REMARK. For definitions of subspace, quotient space, product, coproduct see Hastings [4].

10. Proposition. Let $(X, \xi)$ and $(Y, \eta)$ be heminearness spaces, then

(1) $(X, \xi)$ is a subspace of $(Y, \eta)$ if and only if there exists an injection $f$ : $(X, \xi) \rightarrow(Y, \eta)$ such that $\mathfrak{A} \in \xi$ if and only if $f(\mathfrak{U}) \in \eta$.

(2) $(Y, \eta)$ is a quotient space of $(X, \xi)$ if and only if there exists a surjection $f$ : $(X, \xi) \rightarrow(Y, \eta)$ such that $\mathfrak{A} \in \eta$ if and only if $f^{-1} \mathfrak{A} \in \xi$.

11. Proposition. Let $\left(X_{i}, \xi_{i}\right)_{i \in I}$ be a family of heminearness spaces then

(1) $\left(\prod_{i \in I} X_{i}, \xi\right)$ is the product of this family in $H-N E A R$ if and only if $\xi=\{\mathfrak{U} \subset$ $P\left(\prod_{i \in I} X_{i}\right) \mid \operatorname{Pr}_{i} \mathfrak{A} \in \xi_{i}$ for each $\left.i \in I\right\}$.

(2) $\left(\mathbb{I}_{i \in I} X_{i}, \xi\right)$ is the coproduct of this family in $H-N E A R$ if and only if $\xi=\{\mathfrak{U} \subset$ $P\left(\amalg_{i \in I} X_{i}\right) \mid$ inj $_{i}^{-1} \mathfrak{Q} \in \xi_{i}$ for some $\left.i \in I\right\}$.

Proof. (1) $\xi$ is initial with respect to $\left(\Pi X_{i}, p r_{i},\left(X_{i}, \xi_{i}\right)_{i \in I}\right)$ if and only if

$$
\xi=\cap\left\{\operatorname{Pr}_{i}^{-1}\left(\xi_{i}\right) \mid i \in I\right\}=\left\{\mathfrak{A} \subset P\left(\prod_{i \in I} X_{i}\right) \mid \operatorname{Pr}_{i} \mathfrak{A} \in \xi_{i} \text { for each } i \in I\right\} \text {. }
$$

(2) $\xi$ is final with respect to $\left(\left(X_{i}, \xi_{i}\right), i n j_{i}, \amalg X_{i}\right)_{i \in I}$ if and only if

$$
\begin{aligned}
\xi & =\left(\cup\left\{\operatorname{inj}_{i}\left(\xi_{i}\right) \mid i \in I\right\} \cup\left\{\mathcal{P} \subset P\left(\coprod_{i \in I} X_{i}\right) \mid \cap \mathcal{L} \neq \varnothing\right\}\right)_{n} \\
& =\left(\left\{\mathfrak{U} \subset P\left(\coprod_{i \in I} X_{i}\right) \mid \operatorname{inj}_{i}^{-1} \mathfrak{A} \in \xi_{i} \text { for some } i \in I\right\}\right)_{n} .
\end{aligned}
$$

Let $\beta=\left\{\mathfrak{U} \subset P\left(\amalg_{i \in I} X_{i}\right) \mid\right.$ injj $_{i}^{-1} \mathfrak{U} \in \xi_{i}$ for some $\left.i \in I\right\}$. We claim that $\xi=\beta$. Suppose $\mathrm{Cl}_{\beta} \mathfrak{A} \in \beta$. Then for some $i \in I$, inji $_{i}^{-1}\left(\mathrm{Cl}_{\beta} \mathfrak{A}\right) \in \xi_{i}$. injj $\left(X_{i}\right) \cap$ inj $_{k}\left(X_{k}\right)=\varnothing$ for

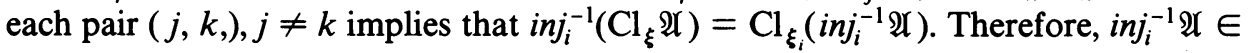
$\xi_{i}$ which implies that $\mathfrak{A} \in \beta$.

12. Proposition. Suppose $(X, \xi)$ and $(Y, \eta)$ are heminearness [ prenearness] spaces and $f:(X, \xi) \rightarrow(Y, \eta)$ is an embedding. Then $f:\left(X, \xi_{s}\right) \rightarrow\left(Y, \eta_{s}\right)$ is an embedding of the nearness [seminearness] space $\left(X, \xi_{s}\right)$ into $\left(Y, \eta_{s}\right)$.

13. Proposition. Let $\left(X_{i}, \beta_{i}\right)_{i \in I}$ be a family of heminearness spaces and let $\xi_{i}=\left(\beta_{i}\right)_{s}$ for each $i \in I$. Let $\left(\prod_{i \in I} X_{i}, \xi\right)$ be the product of $\left(X_{i}, \xi_{i}\right)_{i \in I}$ in NEAR and $\left(\prod_{i \in I} X_{i}, \beta\right)$ the product of $\left(X_{i}, \beta_{i}\right)_{i \in I}$ in $H-N E A R$. Then $\beta_{s}=\xi$.

Proof. $\left(\prod_{i \in I}, \xi\right)$ is the product of $\left(X_{i}, \xi_{i}\right)_{i \in I}$ in $N E A R$ means $\xi=\eta_{s}$ where $\eta=\left\{\mathfrak{A} \subset P\left(\prod_{i \in I} X_{i}\right) \mid P r_{i} \mathfrak{A} \in \xi_{i}\right.$ for each $\left.i \in I\right\}$. ( $\left.\prod_{i \in I} X_{i}, \beta\right)$ is the product of $\left(X_{i}, \beta_{i}\right)_{i \in I}$ in $H-N E A R$ means $\beta=\left\{\mathfrak{A} \subset P\left(\Pi_{i \in I} X_{i}\right) \mid P r_{i} \mathfrak{A} \in \beta_{i}\right.$ for each $\left.i \in I\right\}$. If $\mathfrak{A} \notin \xi$, then there exists $\mathfrak{A}_{1}, \ldots, \mathfrak{A}_{n} \notin \eta$ such that $\mathfrak{A}_{1} \vee \ldots \vee \mathfrak{U}_{n}$ corefines $\mathfrak{A}$ which implies that for each $1 \leqslant i \leqslant n$ there exists $j_{i}$ and $\mathcal{L}_{1}^{j_{i}}, \ldots, \mathcal{L}_{m_{j_{i}}}^{j_{i}} \notin \beta_{j}$ such that $\mathfrak{L}_{1}^{j_{i}} \vee \ldots \vee \mathfrak{Q}_{m_{j_{i}}}^{j_{i}}$ corefines $\operatorname{Pr}_{j} \mathfrak{A}_{i}$. Therefore, $\operatorname{Pr}_{j}^{-1} \mathfrak{L}_{q}^{j_{i}} \notin \beta$ for each $1 \leqslant i \leqslant n$ and for each $1 \leqslant q \leqslant m_{j_{i}}$ and $\bigvee_{i=1}^{n}\left(\bigvee_{q}^{m_{j_{i}}} \mathcal{L}_{q}^{j_{i}}\right)<\mathfrak{U}$. 
14. Remark. H. Herrlich [5] developed a method by which one can construct completions for nearness spaces. Here we imitate the terminology and the construction in order to build completions for heminearness spaces. $H-N E A R$ is the only category containing $N E A R$ known to us where the process of completion can be imitated satisfactorily.

15. Definition. Let $(X, \xi)$ be a heminearness space. The maximal elements of the set $\xi-\{\varnothing\}$, ordered by inclusion are called $\xi$-clusters. A $\xi$-cluster $\mathfrak{A}$ is called a point $\xi$-cluster if and only if there exists $x \in X$ with $\{x\} \in \mathfrak{A}$.

16. Note. If $(X, \xi)$ is a nearness space then a $\xi$-cluster is always a grill in a sense of Chouquet [3], i.e., $A \cup B \in \mathfrak{A}$ if and only if $A \in \mathfrak{A}$ or $B \in \mathfrak{A}$. But $\xi$-cluster in a heminearness space generally fails to be a grill.

EXAMPLE. Let $(\mathbf{R}, \xi)$ be a heminearness space defined as in Example 3(a) and let $x \in R$. Then $e(x)=\{A \subseteq R \mid x \in \mathrm{Cl} A\}$ is a $\xi$-cluster. Let $a<x<b$ and $A=$ $(-\infty, a), B=(b, \infty)$. Then $A \cup B \in e(x)$, but $A \notin e(x)$ and $B \notin e(x)$.

17. Proposition. If $(X, \xi)$ is a heminearness space and if $x \in X$, then $e(x)=\{A$ $\left.\subset X \mid x \in \mathrm{Cl}_{\xi} A\right\}$ is a point $\xi$-cluster. Conversely, any point $\xi$-cluster is of this form.

18. Definition. A heminearness space is called complete if and only if every $\xi$-cluster is a point $\xi$-cluster.

19. Definition. A prenearness space $(X, \xi)$ is called a $T 1$-prenearness space if and only if for any pair of elements $(x, y)$ of $X$, if $\{(x),(y)\} \in \xi$ then $x=y$.

20. THEOREM. Let $(X, \xi)$ be a heminearness space, let $X^{*}$ be the set of all $\xi$-clusters, let $\xi^{*}$ be the set of all $\Omega \subset P X^{*}$ with $(\cup\{\cap \omega \mid \omega \in \Omega\}) \in \xi$ and let $f: X \rightarrow X^{*}$ be the function which maps each $x \in X$ to the point $\xi$-cluster $e(x)$. Then the following hold:

(1) $\left(X^{*}, \xi^{*}\right)$ is a complete T1-heminearness space.

(2) $f:(X, \xi) \rightarrow\left(X^{*}, \xi^{*}\right)$ is a nearness preserving map.

Moreover the following hold:

(a) $\mathfrak{A} \in \xi$ if and only if $f \mathfrak{A} \in \xi^{*}$.

(b) $\mathrm{Cl}_{\xi^{*}} f X=X^{*}$.

(3) $f$ is injective if and only if $(X, \xi)$ is a T1-heminearness space.

(4) $f$ is surjective if and only if $(X, \xi)$ is complete.

21. REMARK. The above construction $f:(X, \xi) \rightarrow\left(X^{*}, \xi^{*}\right)$ is called the completion of $(X, \xi)$.

The proof of Theorem 20 is similar to that of Theorem 5.7 of [4'] and will therefore be omitted.

Note. To show that $f: X \rightarrow X^{*}$ is nearness preserving, we need axiom (N5). This process of completion cannot be imitated for P-NEAR or $S-N E A R$.

Proof of Note. If $\mathfrak{U} \in \xi$ and $B \in \cup\{\cap \omega \mid \omega \in f(\mathfrak{U})\}$, then $B \in \cap f A$ for some $A \in \mathfrak{A}$ which implies that $A \subset \mathrm{Cl}_{\xi} B$. Therefore by $(\mathrm{N} 1)\left\{\mathrm{Cl}_{\xi}(\cup\{\cap \omega \mid \omega \in f(\mathfrak{U})\})\right\}$ $\in \xi$ and by (N5) $\cup\{\cap \omega \mid \omega \in f(\mathfrak{A})\} \in \xi$, i.e., $f(\mathfrak{A}) \in \xi^{*}$.

II. Closure heminearness spaces. We will now introduce the concept of preclosure, semiclosure, and hemiclosure, and define the category $C-H-N E A R$ of closure heminearness spaces. We prove that $C-H-N E A R$ is bicoreflective in $H-N E A R$ and is isomorphic to the category of hemiclosure spaces and continuous functions. 
In the 1973 Categorical Topology Conference at Southern Illinois University in Carbondale, H. Herrlich [7] posed the following question: how far removed is $N E A R$ from TOP? The analogous question in the category of heminearness spaces is: how far removed is $C-H-N E A R$ from $H-N E A R$ ? We will show that any heminearness space is a subspace of some closure heminearness space. Our method can also be used to obtain analogous results in $P-N E A R$. For the case of $S-N E A R$ we proved [4] that any seminearness space can be embedded as a subspace of a product of grill-determined spaces.

22. Definitions. Let $X$ be a set. $\mathrm{Cl}$ an operator on $X$ and consider the following axioms. For any pair $(x, y)$ of elements of $X$ and for any pair $(A, B)$ of subsets of $X$ :

(0) If $x \in \mathrm{Cl}\{y\}$, then $y \in \mathrm{Cl}\{x\}$.

(1) $\mathrm{Cl} \varnothing=\varnothing$.

(2) $A \subset \mathrm{Cl} A$.

(3) If $A \subset B$, then $\mathrm{Cl} A \subset \mathrm{Cl} B$.

(4) $\mathrm{Cl}(A \cup B)=\mathrm{Cl} A \cup \mathrm{Cl} B$.

(5) $\mathrm{ClCl} A=\mathrm{Cl} A$.

$(X, \mathrm{Cl})$ is called a preclosure [semiclosure, hemiclosure, topological] space if and only if $\mathrm{Cl}$ satisfies axioms (0)-(3) [(0)-(4), (0)-(3) and (5), (0)-(5)].

If $\left(X, \mathrm{Cl}_{X}\right)$ and $\left(Y, \mathrm{Cl}_{Y}\right)$ are preclosure spaces, then a function $f:\left(X, \mathrm{Cl}_{X}\right) \rightarrow$ $\left(Y, \mathrm{Cl}_{Y}\right)$ is called continuous if and only if $f\left(\mathrm{Cl}_{X}\right) \subset \mathrm{Cl}_{Y} f A$ for each $A \subset X$.

The category of preclosure [semiclosure, hemiclosure, topological] spaces and continuous functions is called P-CLOS [S-CLOS, H-CLOS, TOP]. Throughout this paper we require that topological spaces satisfy the symmetry axiom (0) above.

22'. REMARK. $S$-CLOS has been studied by E. Čech [2].

23. Definition. A heminearness space $(X, \xi)$ is called a closure heminearness space if and only if it satisfies the following condition:

(c) if $\mathfrak{A} \in \xi$ then $\cap \mathrm{Cl}_{\xi} \mathfrak{A} \neq \varnothing$.

Note that this condition is equivalent to

(c') $\mathfrak{A} \in \xi$ if and only if $\cap \mathrm{Cl}_{\xi} \mathfrak{A} \neq \varnothing$.

24. THEOREM. The full subcategory $C-H-N E A R$ of $H-N E A R$ whose objects are closure heminearness spaces is:

(a) bicoreflective in $H$-NEAR. $1_{X}:\left(X, \xi_{t}\right) \rightarrow(X, \xi)$ is the C-H-NEAR coreflection, where $\xi_{t}=\left\{\mathfrak{A} \subset P(X) \mid \cap \mathrm{Cl}_{\xi} \mathfrak{A} \neq \varnothing\right\}$.

(b) isomorphic to the category $H$-CLOS of hemiclosure spaces and continuous functions. $F: C-H-N E A R \rightarrow H-C L O S$ is an isomorphism, where $F(X, \xi)=\left(X, \mathrm{Cl}_{\xi}\right)$.

25. Proposition. If $\left(X_{i}, \xi_{i}\right)_{i \in I}$ is a family of heminearness spaces, then $\left(X_{i}, \xi_{i}\right)_{i \in I}$ is a family of closure heminearness spaces if and only if $\left(\prod_{i \in I} X_{i}, \xi\right)$, the product of this family in $H-N E A R$ is a closure heminearness space.

Proof. Let $\mathfrak{A} \in \xi$, then for each $i \in I$ there exists $x_{i} \in \cap \mathrm{Cl}_{\xi_{i}} \operatorname{Pr}_{i} \mathfrak{A}$. Let $x \in$ $\Pi_{i \in I} X_{i}$ such that $\operatorname{Pr}_{i} x=x_{i}$ for each $i \in I$, then $x \in \cap \mathrm{Cl}_{\xi} \mathfrak{A} \neq \varnothing$. Suppose there exists $i \in I$ such that $\left(X_{i}, \xi_{i}\right)$ is not a closure heminearness space, i.e., there exists 
$\mathfrak{U} \in \xi_{i}$ such that $\cap \mathrm{Cl}_{\xi_{i}} \mathfrak{U}=\varnothing$. For each $A \in \mathfrak{U}$ let $B_{A} \subset \prod_{i \in I} X_{i}$ such that $\operatorname{Pr}_{i} B_{A}=$ $A$ and $\operatorname{Pr}_{j} B_{A}=X_{j}$ for $j \neq i: i, j \in I$. Let $\mathcal{L}=\left\{B_{A} \mid A \in \mathfrak{X}\right\}$, then $\mathcal{L} \in \xi$ and $\cap \mathrm{Cl}_{\xi} \mathcal{L}$ $=\varnothing$. Therefore, $\left(\prod_{i \in I} X_{i}, \xi\right)$ is not a closure heminearness space.

26. TheOREM. Any heminearness space can be embedded as a subspace of a closure heminearness space.

Proof. Let $(X, \xi)$ be a heminearness space. Let $\alpha=\left\{\mathfrak{U} \in \xi \mid \cap \mathrm{Cl}_{\xi} \mathfrak{A} \neq \varnothing, \mathfrak{U}\right.$ is a stack (i.e., if $A \in \mathfrak{U}$ and $A \subset B$ then $B \in \mathfrak{U}$ ) and $\mathrm{Cl}_{\xi} A \in \mathfrak{U}$ implies that $\left.A \in \mathfrak{U}\right\}$. For each $\mathfrak{A} \in \alpha$, adjoin $x_{\mathfrak{A}}$ to $X$, i.e., let $X^{\prime}=X \cup\left\{x_{\mathfrak{U}} \mid \mathfrak{A} \in \alpha\right\}$. For each $A \subset X^{\prime}$ define $\mathrm{Cl} A=A \cup \mathrm{Cl}_{\xi}(A \cap X) \cup\left\{x_{\mathfrak{A}} \mid \mathfrak{A} \in \alpha\right.$ and $\left.A \cap X \in \mathfrak{A}\right\}$. We claim that $\left(X^{\prime}, \mathrm{Cl}\right)$ is a hemiclosure space. The only nontrivial fact in establishing this claim is that $\mathrm{ClCl} A \subset \mathrm{Cl} A$ for any $A \subset X^{\prime}$.

Let $x \in \mathrm{ClCl} A=\mathrm{Cl} A \cup \mathrm{Cl}_{\xi}((\mathrm{Cl} A) \cap X) \cup\left\{x_{\mathfrak{A}} \mid \mathfrak{A} \in \alpha\right.$ and $(\mathrm{Cl} A) \cap X \in$ If $\}$. Since $(\mathrm{Cl} A) \cap X=\mathrm{Cl}_{\xi}(A \cap X)$ one of the following must hold:

(1) $x \in \mathrm{Cl} A$.

(2) $x \in \mathrm{Cl}_{\xi}\left(\mathrm{Cl}_{\xi}(A \cap X)\right)=\mathrm{Cl}_{\xi}(A \cap X) \subset \mathrm{Cl} A$, i.e., $x \in \mathrm{Cl} A$.

(3) $x=x_{\mathfrak{U}}$, where $\mathfrak{U} \in \alpha$ and $\mathrm{Cl}_{\xi}(A \cap X) \in \mathfrak{U}$ which implies that $A \cap X \in \mathfrak{A}$, i.e., $x \in\left\{x_{\mathfrak{U}} \mid \mathfrak{A} \in \alpha\right.$ and $\left.A \cap X \in \mathfrak{A}\right\} \subset \mathrm{Cl} A$, i.e., $x \in \mathrm{Cl} A$. This completes the proof of $\mathrm{ClCl} A \subset \mathrm{Cl} A$ for any $A \subset X^{\prime}$. Define $\xi^{\prime}=\left\{\mathfrak{U} \subset P\left(X^{\prime}\right) \mid \cap \mathrm{Cl} \mathfrak{A} \neq \varnothing\right\}$, then $\mathrm{Cl}_{\xi^{\prime}} A=\mathrm{Cl} A$ for each $A \subset X^{\prime}$ and $\left(X^{\prime}, \xi^{\prime}\right)$ is a heminearness space. Now, $\mathfrak{U} \in \xi^{\prime}$ if and only if $\cap \mathrm{Cl} \mathfrak{U}=\cap \mathrm{Cl}_{\xi^{\prime}} \mathfrak{U} \neq \varnothing$ which implies that $\left(X^{\prime}, \xi^{\prime}\right)$ is a closure heminearness space. We claim that the inclusion map $i:(X, \xi) \rightarrow\left(X^{\prime}, \xi^{\prime}\right)$ is an embedding. In establishing this claim we will only give the details in the proof of the case where $\mathfrak{A} \in \xi$ and $\cap \mathrm{Cl}_{\xi} \mathfrak{A}=\varnothing$. In this case let $\mathscr{L}=\{B \subset X \mid$ there exists $A \in \mathfrak{U}$ such that $\left.\mathrm{Cl}_{\xi} A \subset \mathrm{Cl}_{\xi} B\right\}$. Then $\mathfrak{A} \subset \mathcal{E}$ and $\mathcal{E} \in \boldsymbol{\alpha}$ which implies that $x_{\mathfrak{L}} \in$ $\cap \mathrm{Cl}_{\xi^{\prime}} \mathcal{L} \subset \mathrm{Cl}_{\xi^{\prime}}, \mathfrak{U} \neq \varnothing$ and so $i(\mathfrak{U})=\mathfrak{A} \in \xi^{\prime}$.

27. REMARK. The construction used in this theorem results in a dense embedding, i.e., $\mathrm{Cl}_{\xi^{\prime}} X=X^{\prime}$.

30. Corollary. The epireflective hull of C-H-NEAR in H-NEAR is H-NEAR.

31. Corollary. Any nearness space can be embedded as a subspace of a nearness space $\left(X^{\prime}, \beta_{s}\right)$ where $\left(X^{\prime}, \beta\right)$ is a closure heminearness space.

We now bring together the results of $\S \S I$ and II.

32. Proposition. Each closure heminearness space is complete.

\section{REFERENCES}

1. H. L. Bentley, Nearness spaces and extensions of topological spaces, Studies in Topology, Academic Press, New York, 1975.

2. E. Čech, Topological spaces, Interscience, New York, 1966.

3. G. Choquet, Sur les notions de filtre et de grille, C. R. Acàd. Sci. Paris Sér. A-B. 224 (1947), $171-173$.

4. M. S. Hastings, Epireflective Hulls in Near, Rev. Roumaine Math. Pures Appl. 1981.

4'. H. Herrlich, A concept of nearness, General Topology Appl. 4 (1974), 191-212.

5. __ Topological structures, Math. Centre Tracts 52 (1974).

6. Cartesian closed topological categories, Math. Colloq. Univ. Cape Town 9 (1974).

7. Remarks and problems concerning nearness structures, Problems in Categorical Topology, (Categorical Topology Conf. at Southern Illinois Univ., Carbondale, 1973). 
8. H. Herrlich and G. E. Strecker, Category theory, Allyn \& Bacon, Boston, Mass., 1973.

9. W. N. Hunsaker and P. L. Sharma, Nearness structures compatible with a topological space, Arch. Math. (Basel) 25 (1974), 172-178.

10. J. R. Isbell, Uniform spaces, Math. Surveys, vol. 12, Amer. Math. Soc., Providence, R.I., 1964.

11. M. Katětov, Convergence structures, General Topology and its Relations to Modern Analysis and Algebra II, Academia, Prague, 1967, pp. 207-216.

12. __ On continuity structures and spaces of mappings, Comment. Math. Univ. Carolinae 6 (1965), 257-278.

13. L. D. Nel, Initially structured categories and Cartesian closedness, Canad. J. Math. 27 (1975), 1361-1377.

14. W. A. Robertson, Convergence as a nearness concept, Dissertation, Carleton University, 1975.

15. N. A. Shanin, On separation in topological spaces, Dokl. Akad. Nauk SSSR 38 (1943), 110-113.

Department of Mathematics, William Paterson College, Wayne, New Jersey 07470

Current address: Department of Mathematics, Marymount College, Tarrytown, New York 10591 